\title{
Screening of potential gene markers for predicting carotid atheroma plaque formation using bioinformatics approaches
}

\author{
GUIMING WANG $^{1 *}$, DONG KUAI $^{2 *}$, YUDONG YANG $^{1}$, GAOCHAO YANG $^{1}$, ZHIGANG WEI $^{1}$ and WENBO ZHAO ${ }^{1}$ \\ Departments of ${ }^{1}$ Surgery and ${ }^{2}$ Neurosurgery, \\ The First Hospital of Shanxi Medical University, Taiyuan, Shanxi 030001, P.R. China
}

Received January 20, 2016; Accepted January 12, 2017

DOI: $10.3892 / \mathrm{mmr} .2017 .6273$

\begin{abstract}
The present study aimed to investigate potential gene markers for predicting the formation of carotid atheroma plaques using high-throughput bioinformatics methods. The GSE43292 gene expression profile was downloaded from the Gene Expression Omnibus database.Following data processing, differentially expressed genes (DEGs) were screened using a paired t-test in the Linear Models for Microarray Data package with the criteria of a false discovery rate of $\mathrm{P}<0.05$ and $\log 2$ fold-changel $\geq 0.58$, followed by functional enrichment, protein-protein interaction (PPI) network construction, key node and module analysis, and prediction of transcription factors (TFs) targeting genes in the significant modules. The results revealed that the gene expression profiles from 32 paired samples of carotid atheroma plaque tissue and macroscopically intact tissue were obtained, based on which 886 DEGs, including 513 upregulated genes and 373 downregulated genes, were identified. The upregulated and downregulated gene sets were enriched in 24 and 13 pathways, respectively. The PPI network constructed with these DEGs comprised 35 key nodes with degrees $\geq 20$, among which spleen tyrosine kinase $(S Y K), L Y N$ and phosphatidylinositol-4,5-bisphosphate 3-kinase catalytic subunit $\gamma(P I K 3 C G)$ were the three highest. A significant module was mined in the PPI network, which consisted of 29 DEGs targeted by 11 TFs. The DEGs between the carotid atheroma plaque and macroscopically intact tissue samples may be involved in carotid atherogenesis. Key nodes in the PPI network constructed from these DEGs and the genes involved in the significant module, including $S Y K, L Y N$ and $P I K 3 C G$, are promising for the prediction of carotid plaque formation.
\end{abstract}

Correspondence to: Dr Zhigang Wei or Dr Wenbo Zhao, Department of Surgery, The First Hospital of Shanxi Medical University, 85 Jiefang South Road, Taiyuan, Shanxi 030001, P.R. China

E-mail: weizhigangwz@hotmail.com

E-mail: 1376510049@qq.com

${ }^{*}$ Contributed equally

Key words: carotid atheroma, differentially expressed genes, protein-protein interaction network, gene marker

\section{Introduction}

Strokes are reported to be the third leading cause of mortality following cancer and heart disease, and the most common cause of adult disability in developed countries (1). It is estimated that $85 \%$ of strokes can be ascribed to atherosclerotic disease, and $10 \%$ are specifically associated with carotid atheroma $(2,3)$. Several factors have been identified to predispose to carotid atherosclerosis, including male gender, advancing age, hypercholesterolemia, systolic hypertension and obesity (4). However, these classic characteristics are generally poor at predicting the risk of thromboembolism, which may lead to $80 \%$ needlessly exposed to the surgical risks of carotid endarterectomy (5). Thus, it is important to investigate markers with improved ability in predicting the formation of carotid atheroma plaques.

With the emergence and improvement of gene microarray technology, global mRNA expression has been investigated for screening mRNA populations, which are differentially regulated in varied disease processes, and to provide clues to the underlying molecular pathology. In terms of carotid atheroma, a microarray study by Ayari and Bricca (6) was performed in patients with significant carotid stenosis, and used for comparing gene expression between carotid plaque and intact arterial tissues. Expression profiling provides substantial valuable information, although the previous study focused on the gene expression of CD163 and HO-1 only, which suggested more pronounced induction of atheromatous plaque formation with these two genes. The deep mining of this set of data is urgently required as it is likely to assist in screening potential gene markers of carotid atheroma plaque formation.

In the present study the genome-wide expression profile of human carotid atheroma were analyzed comprehensively using high-throughput bioinformatics methods. Differentially expressed genes (DEGs) were identified in the carotid atheroma plaque and compared with the macroscopically intact carotid tissue adjacent to the atheroma plaque. Functional annotation and pathway enrichment were then performed, followed by the construction of protein-protein interaction (PPI) networks, analysis of key nodes and prediction of transcription factors (TFs). The aim of the present study was to examine potential gene markers for predicting the formation of carotid atheroma plaques. 


\section{Materials and methods}

Data source. The GSE43292 gene expression profile and its corresponding platform annotation files were downloaded from the Gene Expression Omnibus (GEO) database (http://www. ncbi.nlm.nih.gov/geo/) (7). This data set was submitted by Ayari and Bricca (6) on 4th January 2013, last updated on 21st May 2015 and stockpiled on the GPL6244 platform (HuGene-1_0-st) Affymetrix Human Gene 1.0 ST Array [transcript (gene) version]). This gene expression data consisted of 32 paired samples of carotid atheroma plaque and macroscopically intact tissue adjacent to the atheroma plaque. Each paired sample was collected from sections obtained through carotid endarterectomy in one hypertensive patient. Specifically, the samples of the atheroma plaque were at stage IV or above according to the Stary classification (8), and contained the core and shoulders of the plaque, whereas the other group of samples were distant macroscopically intact tissue at stages I and II.

Data preprocessing. For the optimal mining of essential information from the gene expression profile, the raw level data were first preprocessed using the Robust Multi-array Average method in the Bioconductor oligo package (version 2.1; http://www. bioconductor.org), which is based on the Bioconductor principles of reproducibility, transparency and efficiency of development (9). The probe ID for each gene was then converted to a gene symbol using the hugene10sttranscriptcluster.db, org. Hs.eg.db and annotate package in Biocondctuor (http://www. bioconductor.org/). For gene symbols corresponding to multiple probes IDs, the mean of these probes was calculated as the representative expression level of this gene.

Screening for DEGs. To identify the DEGs between the carotid atheroma plaque and macroscopically intact carotid tissue samples, a paired $t$-test in the Linear Models for Microarray Data (LIMMA) package of R/Bioconductor software was performed for analyzing data from the gene expression experiments (10). The Benjamini-Hochberg methods (11) were further used to correct the P-value, and a false discovery rate of the P-value (FDP) was calculated (12). FDP $<0.05$ accompanied with $\mid \log _{2}$ fold-change $(\mathrm{FC}) \mid \geq 0.58$ were considered the thresholds for the identification of DEGs, which were grouped as upregulated and downregulated genes.

Functional annotation and pathway enrichment. The Database for Annotation Visualization and Integrated Discovery (DAVID; http://david.abcc.ncifcrf.gov) is primarily used for functional annotation and classification. This tool was designed to systematically extract biological meanings from a large gene or protein list through a novel agglomeration algorithm (13). To investigate the potential functions of the DEGs screened out above, DAVID was used to map the upregulated and downregulated genes to Gene Ontology (GO) terms and the Kyoto Encyclopedia of Genes and Genomes (KEGG) pathways, respectively. The GO is determined using the Gene Ontology Consortium (http://www.geneontology.org) and widely used to produce a dynamic and controlled vocabulary for all eukaryotes with accumulation and alterations in gene and protein roles in cells (14). To describe gene product attributes, GO provides three structured networks of defined terms, including cellular compartment (CC), biological process (BP) and molecular function (MF). By contrast, the KEGG pathway database (http://www.genome.ad.jp/kegg) contains information on how molecules or genes are networked, which is essentially a combined map of $\sim 120$ existing pathway maps (15). In the present study, GO terms with a threshold of $\mathrm{P}<0.05$, and KEGG pathways with a threshold of $\mathrm{P}<0.05$ and enriched gene count $\geq 3$ were selected.

Construction of the PPI network. PPIs are considered to be important for understanding the potential functions of a certain protein, and the Search Tool for the Retrieval of Interacting Genes (STRING; http://string-db.org/) is designed to provide such a global perspective based on criteria, including experimental evidence, neighborhood, gene fusion, co-occurrence, co-expression, existing databases and text-mining (16) In the present study, PPI pairs were predicted using STRING, and those with high confidence (PPI score $>0.07$ ) were selected for construction of the PPI network. The PPI network was visualized using Cytoscape 2.8 (http://cytoscape.org/) (17), followed by module analyses using ClusterONE with the threshold of $<4 \mathrm{e}-4$. The genes in the significant modules were further mapped to KEGG pathways for functional analysis.

Functional analysis for key nodes in the PPI network. Functional analyses were performed for key nodes in the PPI network constructed above. GenCLiP 2.0 is a web-based text-mining server, which can be used for the analysis of human genes with enriched keywords and molecular interactions (http://ci.smu.edu.cn/GenCLiP2.0/confirm_keywords. php). The Gene Cluster with Literature Profiles module in GenCLiP can annotate the input genes by generating statistically over-represented keywords based on the occurrence frequencies of free terms in gene-based literature. In addition, Agilent Literature Search software is a meta-search tool for automatically querying multiple text-based search engines to identify and extract associations among genes/proteins of interest (http://www.agilent.com/labs/research/litsearch.html). In the present study, the potential functions of key nodes in the PPI network were predicted using the Gene Cluster with Literature Profiles module with a threshold of $\mathrm{P} \leq 0.05$ and Hit $\geq 6$, and their associations with the formation of atheroma plaques were identified using Agilent Literature Search software with the key word 'atheroma plaque'.

Prediction of TFs. iRegulon, as a Cytoscape plugin, was developed to reverse-engineer the transcriptional regulatory network underlying a co-expressed gene set using cis-regulatory sequence analysis and integrating databases of TFs, including Transfac, Jaspar, Encode, Swissregulon and Homer (18). By setting the minimum identity between orthologous genes as 0.05 and the maximum false discovery rate on motif similarity as 0.001 , iRegulon was used to predict TFs targeting to genes in the modules identified above with a normalized enrichment score $>5$.

\section{Results}

Data processing and DEG screening. The profile normalization is shown in Fig. 1A and B. The median base-line level in 

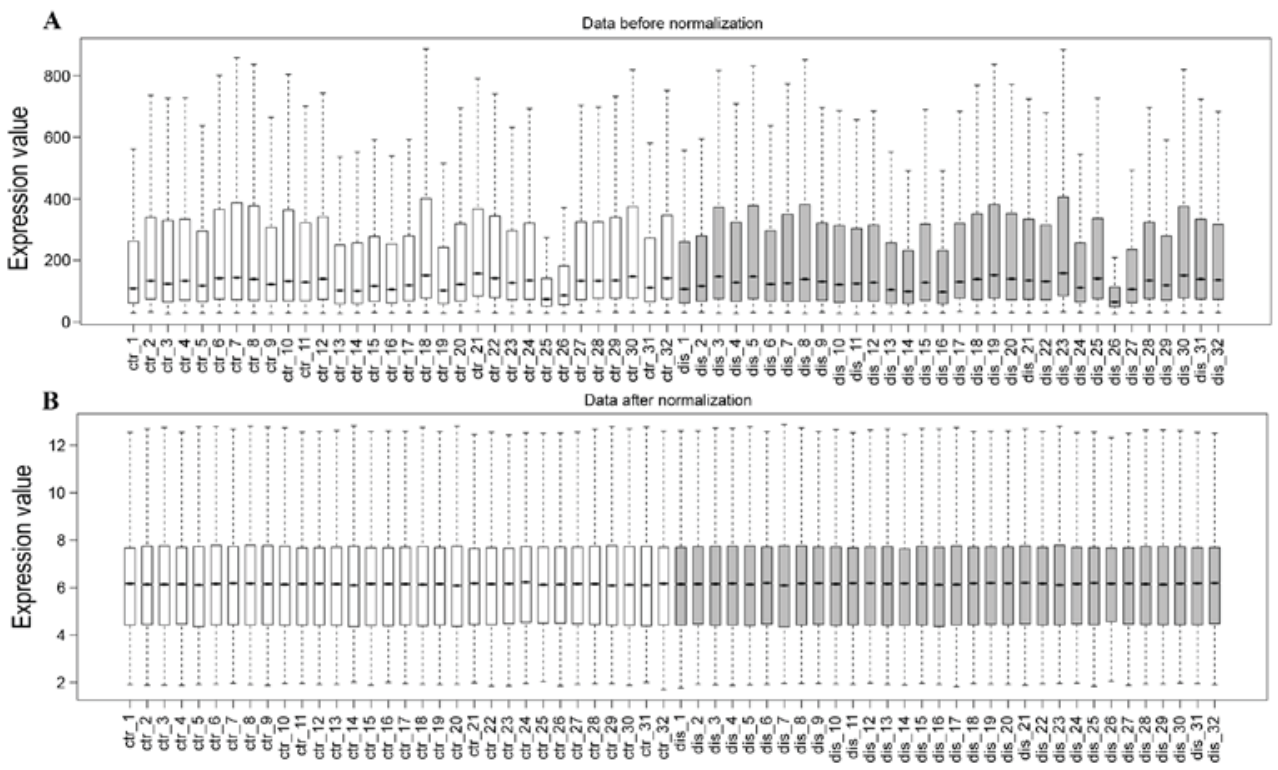

Figure 1. Box plots for the expression profiles of microarray data (A) prior to normalization and (B) following normalization. The box refers to the quartile distribution (25-75\%) range, with the median presented as a central black horizontal line in the box. The interval represents the minimum and maximum values, excluding outliers.

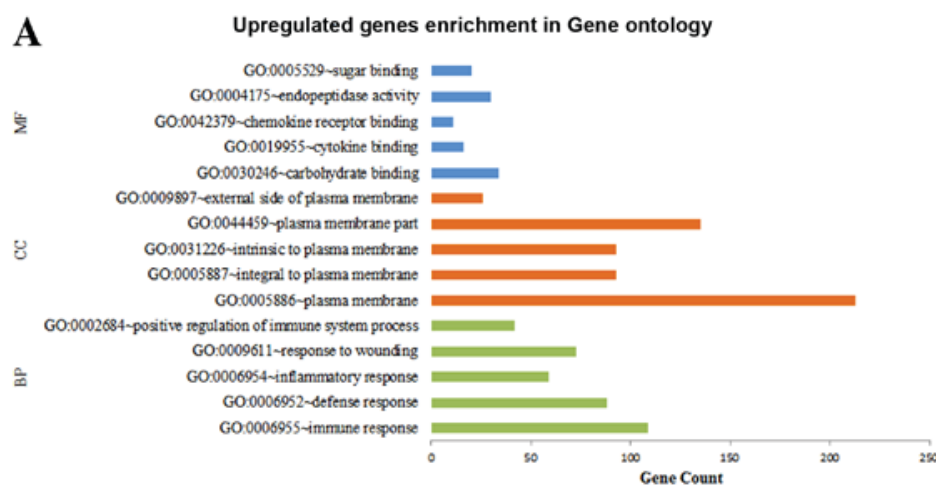

B

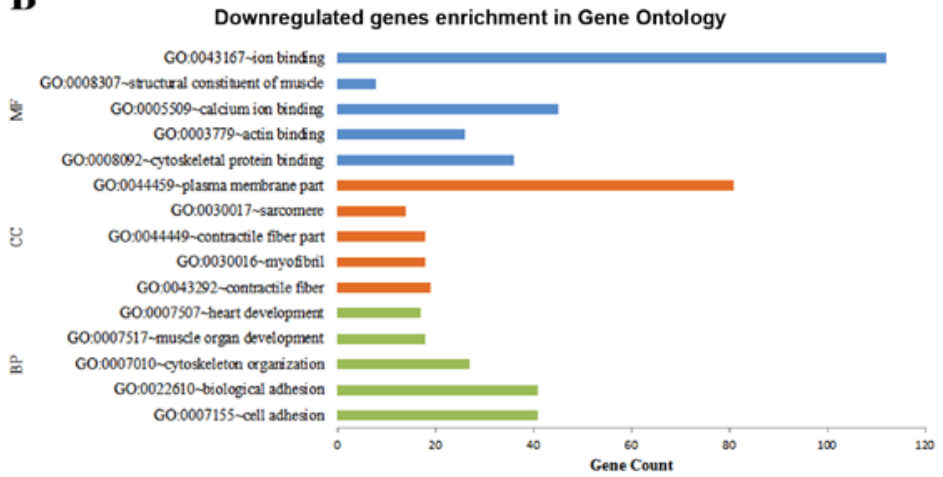

Figure 2. GO analysis. (A) Upregulated genes and (B) downregulated genes. GO, Gene Ontology; MF, molecular function; CC, cellular component; BP, biological process.

the box plot indicates well-effected normalization. With the criteria of $\mathrm{FDP}<0.05$ and $\log 2 \mathrm{FCl} \geq 0.58$, a total of $886 \mathrm{DEGs}$ were finally screened out from the carotid atheroma plaque samples, when compared with those of the macroscopically intact carotid tissue, including 513 upregulated genes and 373 downregulated genes.
Functional enrichment of DEGs. DAVID was used for predicting the potential functions of the DEGs by mapping the upregulated and downregulated genes to GO terms and the KEGG database, respectively. The top five significant GO terms of the MF, CC and BP categories enriched by the upregulated and downregulated genes, respectively, are 
A
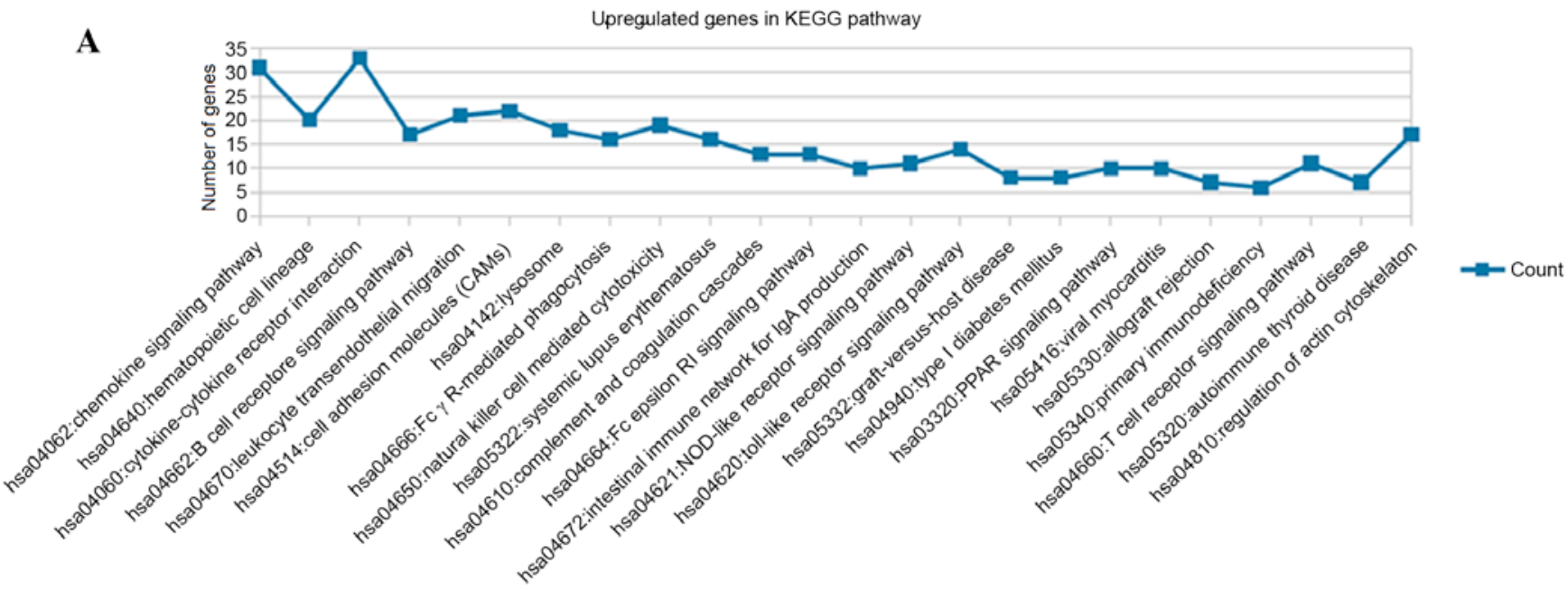

B

Downregulated genes in KEGG pathway

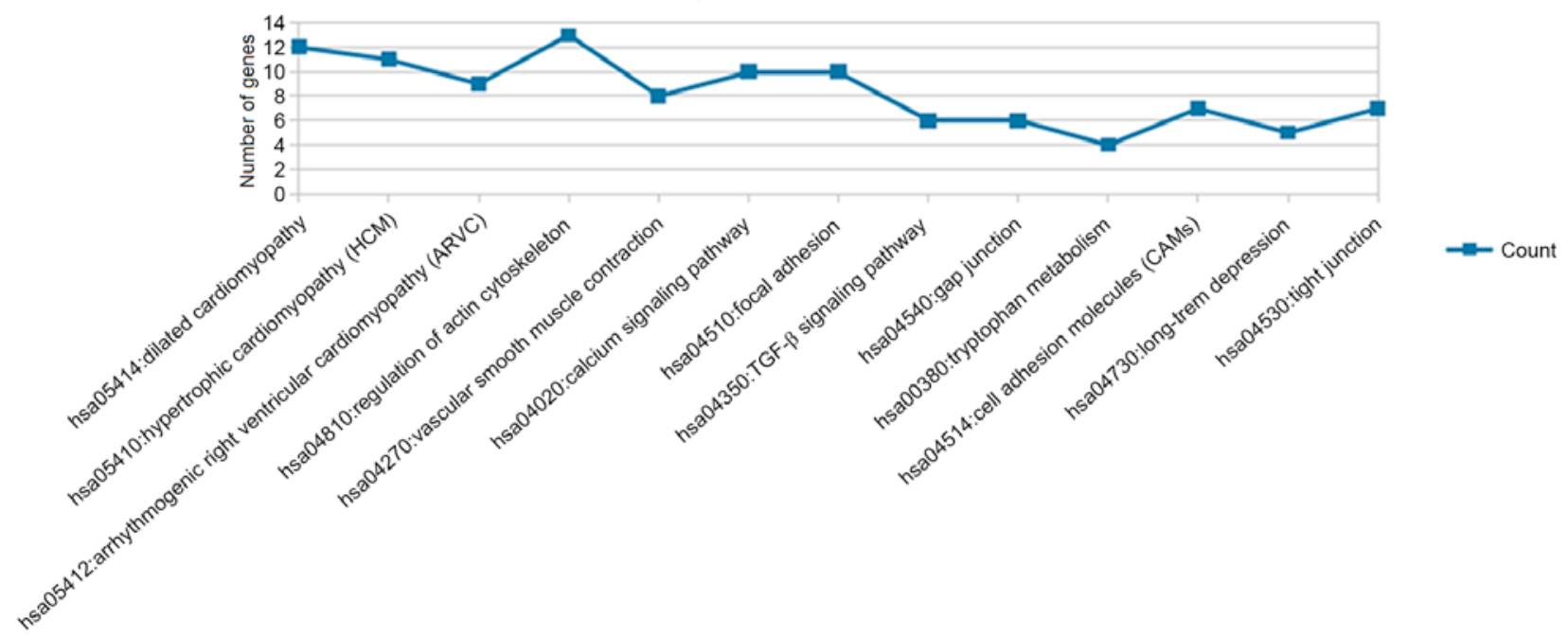

Figure 3. Pathway enrichment analysis. Pathway enrichment for (A) Upregulated genes and (B) downregulated genes.

shown in Fig. 2. The results demonstrated that the upregulated genes were predominantly associated with MFs, including carbohydrate binding, CCs, including plasma membrane and BPs, including immune response (Fig. 2A), whereas the downregulated genes were predominantly involved in MFs, including ion binding, $\mathrm{CCs}$, including plasma membrane part and BPs, including cell adhesion (Fig. 2B). The results for the KEGG pathway enrichment are shown in Fig. 3, which indicated that the upregulated genes were significantly enriched in 24 pathways, including the chemokine signaling pathway and cytokine-cytokine receptor interaction (Fig. 3A); whereas the downregulated genes were predominantly involved in 13 pathways, including dilated cardiomyopathy and regulation of actin cytoskeleton (Fig. 3B).

Construction of PPI network and functional analysis for key genes. The Cytoscape tool visually constructed the PPI network with 478 nodes and 1,457 edges, which were predicted using STRING with a PPI score $>0.07$ (Fig. 4). In the PPI network, 35 nodes with a degree $\geq 20$ were regarded as key genes (Table I), among which only five were downregulated and the others were upregulated in the carotid atheroma plaque samples, compared with the macroscopically intact tissue samples adjacent to the atheroma plaque. Of the 35 key nodes, SYK, LYN and PIK3CG were the top three nodes, with degrees of 43,43 and 37 , respectively. The heat map indicated that these 35 genes were able to distinguish the two groups of tissue samples (Fig. 5). Literature mining using Gene Cluster with Literature Profiles revealed that these key nodes clustered in biological functions, which included phospholipase $\mathrm{C}$, map kinase, cytokine production and inflammatory response (Table II). The disease network was constructed with 296 nodes and 1,118 edges associated with the above 35 genes using Agilent Literature Search software (Fig. 6). Certain genes in this disease network were the DEGs identified above, indicating that these genes may be vital in the formation of atheroma plaques.

Module analysis. In the PPI network, one module was mined using ClusterOne software with a threshold $<4 \mathrm{e}-4$. A total of 29 DEGs, including 22 upregulated and seven downregulated genes, were used to construct this module and 11 TFs were predicted to target these genes by iRegulon (Fig. 7). The KEGG pathway enrichment showed that genes in this module were predominantly involved in the chemokine signaling pathway and cytokine-cytokine receptor interaction (Table III). 


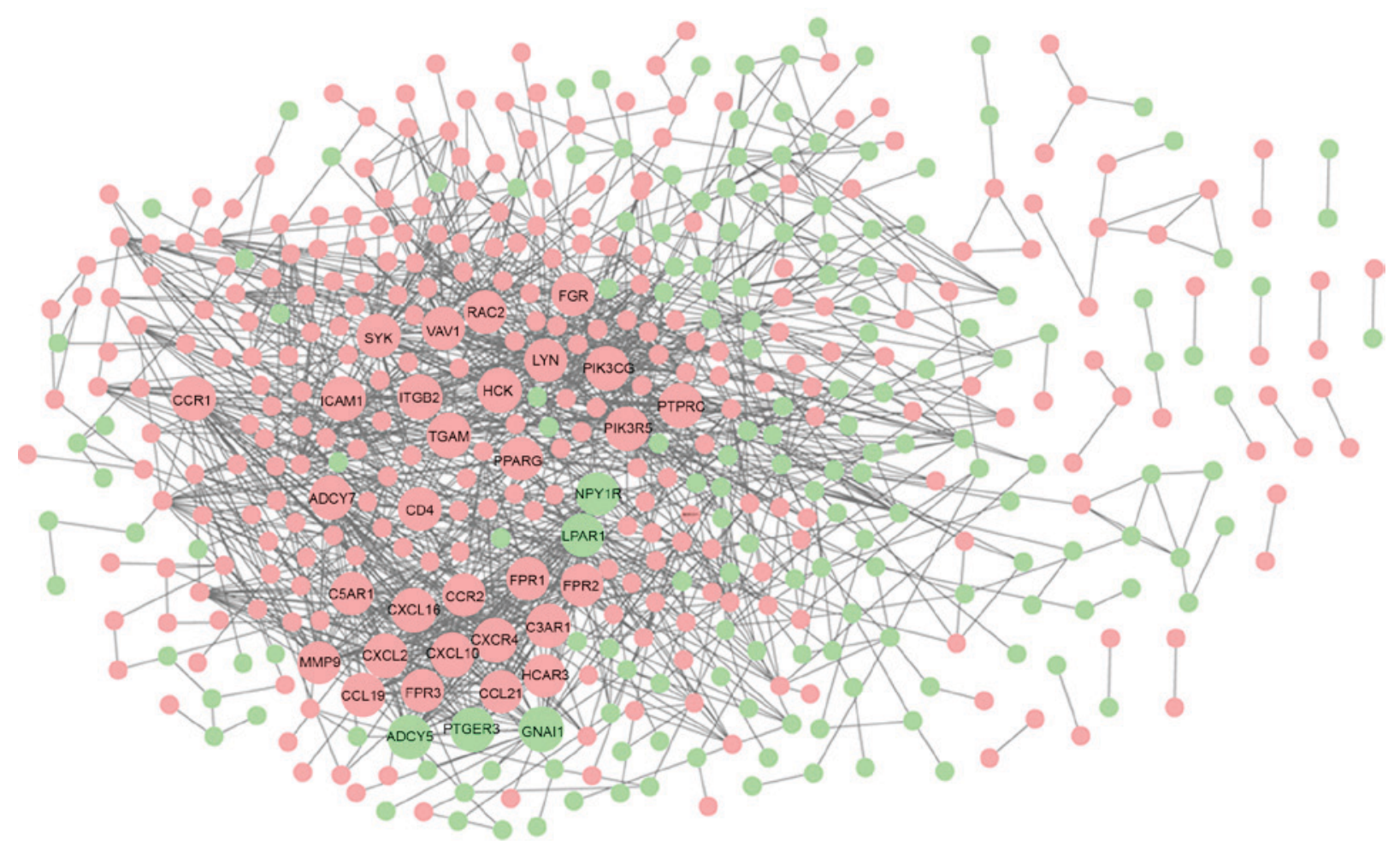

Figure 4. Protein-protein interaction network. Red circles represent upregulated genes and green circle represent downregulated genes. The lines represent the interaction between two nodes.
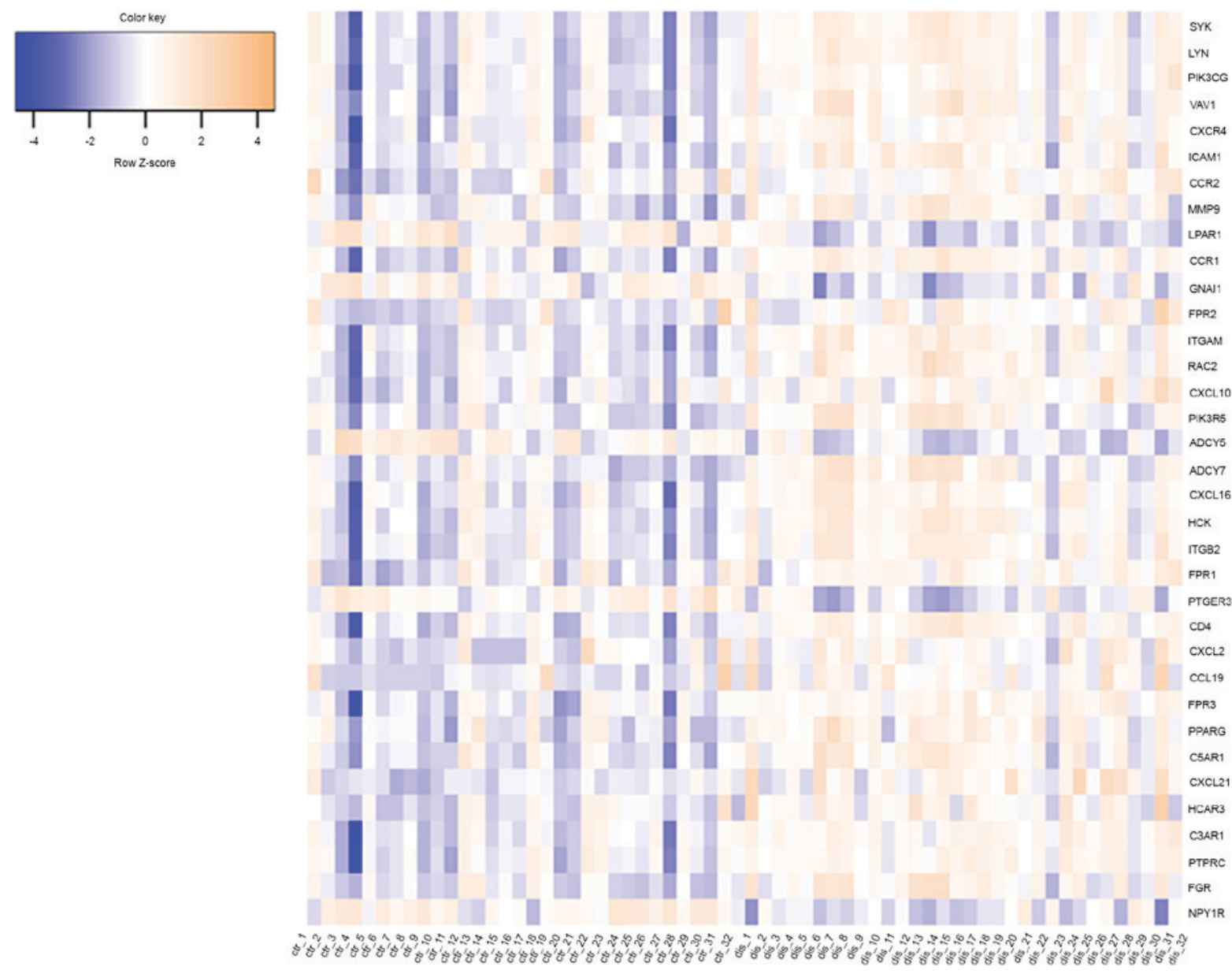

Figure 5. Heat map for key nodes in the protein-protein interaction network. The scaled expression of the predicted key nodes, denoted as the row Z-score, is plotted in with a blue and brown color scale, with blue indicating high expression and sandy-brown indicating low expression. 
Table I. Key nodes in the protein-protein interaction network with degrees $\geq 20$.

\begin{tabular}{lcc}
\hline Gene symbol & Degree & Log $_{2}$ fold-change \\
\hline SYK & 43 & 0.854037 \\
LYN & 43 & 0.797114 \\
PIK3CG & 37 & 0.721574 \\
VAV1 & 34 & 0.712220 \\
CXCR4 & 33 & 0.728104 \\
ICAM1 & 33 & 0.765050 \\
CCR2 & 32 & 0.671559 \\
MMP9 & 31 & 1.817924 \\
LPAR1 & 31 & -0.648530 \\
CCR1 & 30 & 1.177328 \\
GNAI1 & 28 & -0.624960 \\
FPR2 & 28 & 0.708739 \\
ITGAM & 28 & 0.994177 \\
RAC2 & 27 & 0.871698 \\
CXCL10 & 26 & 1.046254 \\
PIK3R5 & 26 & 0.592723 \\
ADCY5 & 25 & -0.936170 \\
ADCY7 & 25 & 0.611163 \\
CXCL16 & 24 & 0.708373 \\
HCK & 24 & 0.789003 \\
ITGB2 & 24 & 0.967159 \\
FPR1 & 23 & 0.753358 \\
PTGER3 & 23 & -0.600980 \\
CD4 & 23 & 0.926358 \\
CXCL2 & 22 & 0.581285 \\
CCL19 & 21 & 0.908325 \\
FPR3 & 21 & 0.815153 \\
PPARG & 21 & 0.689479 \\
C5AR1 & 20.829772 \\
CCL21 & 20 & 0.620375 \\
HCAR3 & 20.580189 \\
C3AR1 & 0.676339 \\
PTPRC & FGR & 0.782351 \\
NPY1R & 0.767073 \\
\hline & -1.286510 \\
\hline
\end{tabular}

\section{Discussion}

Strokes are one of the leading causes of mortality and long-time disability in the majority of countries worldwide (19). Generally, a stroke is caused by an embolus or thrombus arising from a ruptured carotid atheromatous plaque or, more rarely, results from hemodynamic changes induced by the considerable contraction of the carotid lumen (20). Strokes are described as being avoidable, however, a major challenge is identifying efficient markers to detect patients who are at risk of stroke. The present study aimed to investigate gene markers for predicting the formation of atheroma plaques by applying high throughput bioinformatics methods for comprehensive analyses of gene expression data from 32 paired samples of carotid atheroma plaque and macroscopically intact tissue adjacent to the atheroma plaque. A total of 886 DEGs, including 513 upregulated and 373 downregulated genes, were identified. This set of upregulated genes were predicted to be significantly involved in BPs, including carbohydrate binding and immune response, and were involved in 24 pathways. The downregulated genes were predominantly involved in ion binding and cell adhesion via 13 potential pathways. The PPI network constructed using these DEGs revealed 35 key nodes and one significant module. These results provided novel insight and valuable information for improving understanding the pathogenesis of carotid atheroma. Notably, key nodes in the PPI network and genes in the significant modules, including $S Y K, L Y N$ and PIK3CG, are promising for the prediction of carotid atheroma plaque formation.

SYKis a $72 \mathrm{kDa}$ non-receptor tyrosine kinase and its highest level expressed is in hematopoietic cells. SYK contains three functional domains, including two SRC homology 2 domains and a kinase domain (BOX 1). Investigations have focused on SYK as it is a potential therapeutic target in chronic inflammatory diseases, particularly in rheumatoid arthritis and asthma (21). Apoptotic cell accumulation is a major feature of advanced human atherosclerotic lesions and is associated with increased susceptibility to thrombotic plaque complications (22). A previous study reported that defective tyrosine kinase signaling in bone marrow cells may lead to the accumulation of apoptotic cells within atherosclerotic lesions, increase the proinflammatory immune response and accelerate atherosclerosis (23). As a tyrosine kinase, SYK has also been found to be correlated with atherogenesis. Choi et al (24) demonstrated that macrophage responses mediated by SYK may contribute to chronic inflammation in atherosclerosis in humans. Atherosclerotic lesions in low density lipoprotein-deficient mice, were examined following treatment with an SYK inhibitor, and were found to contain fewer macrophages, but more smooth muscle cells and collagen, which are features of more stable plaques in humans (25). The SYK inhibitor, fostamatinib was identified as a potentially beneficial therapeutic strategy for patients with atherosclerosis as it was reported to reduce atherosclerotic lesion size by up to $59.6 \%$ in mice (25). In the present study, the expression of SYK was found to be upregulated in the carotid atheroma plaque samples, compared with macroscopically intact tissue samples from the same hypertensive patient. Functional enrichment analysis revealed that SYK was associated with positive regulation of the immune system process and leukocyte activation, which was in accordance with the previous studies. Of note, SYK was characterized with the highest degree in the PPI network constructed of DEGs between the two sets of samples. Thus, it was hypothesized that SYK is a key factor in the process of carotid atherosclerosis and promising in the prediction of plaque formation.

LYN, also known as FYN, is a $59 \mathrm{kDa}$ protein located on chromosome 6q21. LYN is also a member of the Src family of non-receptor tyrosine kinases. LYN is identified as a convergence point of several signaling pathways, and is vital in a number of BPs, including regulating cell cycle entry, growth, proliferation and cell-cell adhesion (26). Using complementary inhibition strategies, Toubiana et al (27) showed that LYN may be involved in nuclear factor (NF)-кB activation in 
Table II. Functional enrichment of key nodes in the protein-protein interaction network.

\begin{tabular}{llll}
\hline Key word & Hits (n) & P-value & Genes \\
\hline
\end{tabular}

\#cluster1

Enrichment score: 48.42

PHOSPHOLIPASE C

MAP KINASE

$20 \quad 2.08 \mathrm{E}-51$

\#cluster2

Enrichment score: 47.48

CYTOKINE PRODUCTION

9.57E-76

INFLAMMATORY RESPONSE

$2.28 \mathrm{E}-38$

TUMOR NECROSIS FACTOR

$1.68 \mathrm{E}-30$

\#cluster3

Enrichment score: 41.88

CELL ADHESION

CELL ACTIVATION

C REACTIVE PROTEIN

TUMOR NECROSIS FACTOR

HUMAN UMBILICAL VEIN

\#cluster5

Enrichment score: 32.27

MONOCYTE

CHEMOATTRACTANT

PROTEIN

MACROPHAGE

INFLAMMATORY

PROTEIN

CENTRAL NERVOUS

SYSTEM
26

$2.95 \mathrm{E}-62$

7.87E-13

16

$1.57 \mathrm{E}-48$

17

7.91E-54

C5AR1, CCL19, CCL21, CCR1, CCR2, CD4, CXCL10, CXCL16, CXCL2, CXCR4, FPR1, FPR2, HCK, ICAM1, ITGAM, ITGB2, LYN, MMP9, PIK3CG, PPARG, PTPRC, RAC2, SYK, VAV1

C3AR1, C5AR1, CCL19, CCL21, CCR1, CCR2, CD4, CXCL10, CXCL16, CXCL2, CXCR4, FGR, FPR1, FPR2, HCK, ICAM1, ITGAM, ITGB2, LYN, MMP9, PIK3CG, PPARG, PTPRC, RAC2, SYK, VAV1

C5AR1, CCR2, CD4, CXCL10, CXCL16, CXCL2, FPR1, ICAM1, ITGAM, ITGB2, MMP9, PPARG, PTPRC

C5AR1, CCR2, CD4, CXCL10, CXCL16, CXCL2, CXCR4, FGR, FPR1, ICAM1, ITGAM, ITGB2, MMP9, PIK3CG, PPARG, PTPRC

C5AR1, CCR2, CD4, CXCL10, CXCL16, CXCL2, CXCR4, FPR1, FPR2, ICAM1, ITGAM, ITGB2, LPAR1, MMP9, PIK3CG, PPARG, PTPRC

$18 \quad 2.13 \mathrm{E}-26$

$19 \quad 1.87 \mathrm{E}-11$
C5AR1, CCL19, CCL21, CCR1, CCR2, CD4, CXCL10, CXCL16, CXCL2, CXCR4, FPR1, ICAM1, ITGAM, ITGB2, MMP9, PIK3CG, PPARG, PTPRC C5AR1, CCL19, CCL21, CCR1, CCR2, CD4, CXCL10, CXCL16, CXCL2, CXCR4, FPR1, ICAM1, ITGAM, ITGB2, MMP9, PIK3CG, PPARG, PTPRC ADCY5, C3AR1, C5AR1, CCL19, CCL21, CCR1, CCR2, CD4, CXCL10, CXCL2, CXCR4, FPR1, ICAM1, ITGAM, ITGB2, MMP9, PIK3CG, PPARG, PTPRC 
Table II. Continued.

\begin{tabular}{|c|c|c|c|}
\hline Key word & Hits (n) & P-value & Genes \\
\hline CELL ADHESION MOLECULE & 20 & $6.56 \mathrm{E}-45$ & $\begin{array}{l}\text { C5AR1, CCL19, CCL21, CCR1, CCR2, CD4, CXCL10, } \\
\text { CXCL16, CXCL2, CXCR4, FPR1, ICAM1, ITGAM, ITGB2, } \\
\text { MMP9, PIK3CG, PPARG, PTPRC, RAC2, SYK }\end{array}$ \\
\hline TOLL-LIKE RECEPTOR & 21 & $1.87 \mathrm{E}-58$ & $\begin{array}{l}\text { C5AR1, CCL19, CCL21, CCR1, CCR2, CD4, CXCL10, } \\
\text { CXCL16, CXCL2, CXCR4, FPR1, FPR2, ICAM1, ITGAM, } \\
\text { ITGB2, LYN, MMP9, PIK3CG, PPARG, PTPRC, SYK }\end{array}$ \\
\hline \multicolumn{4}{|l|}{ \#cluster6 } \\
\hline \multicolumn{4}{|l|}{ Enrichment score: 28.75} \\
\hline MITOGEN ACTIVATED PROTEIN & 27 & $4.90 \mathrm{E}-39$ & $\begin{array}{l}\text { C3AR1, C5AR1, CCL19, CCL21, CCR1, CCR2, CD4, CXCL10, } \\
\text { CXCL2, CXCR4, FGR, FPR1, FPR2, HCK, ICAM1, ITGAM, } \\
\text { ITGB2, LPAR1, LYN, MMP9, PIK3CG, PPARG, PTGER3, } \\
\text { PTPRC, RAC2, SYK, VAV1, }\end{array}$ \\
\hline SIGNAL TRANSDUCTION & 28 & $6.54 \mathrm{E}-20$ & $\begin{array}{l}\text { C3AR1, C5AR1, CCL19, CCL21, CCR1, CCR2, CD4, CXCL10, } \\
\text { CXCL16, CXCL2, CXCR4, FGR, FPR1, FPR2, HCK, ICAM1, } \\
\text { ITGAM, ITGB2, LPAR1, LYN, MMP9, PIK3CG, PPARG, } \\
\text { PTGER3, PTPRC, RAC2, SYK, VAV1 }\end{array}$ \\
\hline
\end{tabular}

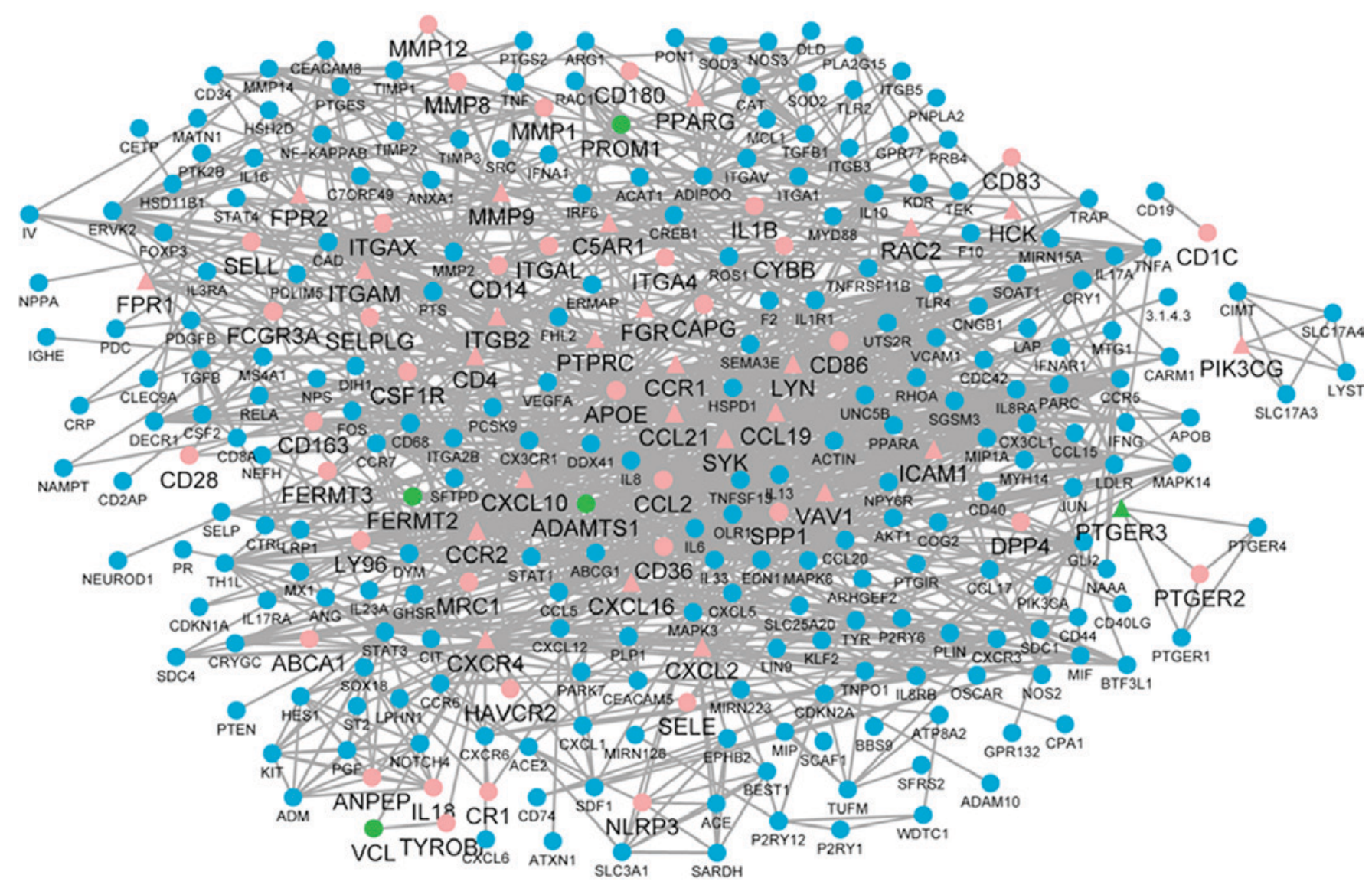

Figure 6. Protein-disease network constructed using Agilent Literature Search. The triangles represent the key nodes in the protein-protein interaction network, whereas pink circles and green circles represent the identified upregulated and downregulated genes, respectively. The blue circles represent other genes that were reported to be associated with this disease in scientific literature and other unstructured text. The grey lines indicate the interactions between two nodes.

human cellular models. Monocyte chemoattractant protein-1, as an inflammatory marker increased with arteriosclerosis, was found to be downregulated by inhibiting the activation of
$\mathrm{NF}-\kappa \mathrm{B}$, which is the vascular protective mechanism underlying the antihypertensive action of nifedipine (28). In the present study, $L Y N$ was found to be differentially expressed in the 
Table III. Kyoto Encyclopedia of Genes and Genomes pathway enrichment of genes in the significant module.

\begin{tabular}{|c|c|c|c|}
\hline Term & $\mathrm{n}$ & P-value & Genes \\
\hline hsa04062: Chemokine signaling pathway & 17 & $2.23 \mathrm{E}-17$ & $\begin{array}{l}\text { ADCY7, GNAI1, ADCY5, CCR1, CXCL2, CCL19, } \\
\text { CCL8, CCL18, CXCL10, CCL13, ARRB2, CXCR4, } \\
\text { ARRB1, CCL21, CXCL16, CCR2, GRK5 }\end{array}$ \\
\hline hsa04060: Cytokine-cytokine receptor interaction & 11 & 2.84E-07 & $\begin{array}{l}\text { CCL13, CCL21, CXCR4, CCR1, } \\
\text { CXCL16, CXCL2, CCR2, CCL8, CCL19, CCL18, CXCL10 }\end{array}$ \\
\hline hsa04080: Neuroactive ligand-receptor interaction & 8 & $2.18 \mathrm{E}-04$ & $\begin{array}{l}\text { C3AR1, C5AR1, PTGER3, FPR1, FPR3, FPR2, } \\
\text { NPY1R, LPAR1 }\end{array}$ \\
\hline hsa04540: Gap junction & 4 & 0.010074 & ADCY7, GNAI1, ADCY5, LPAR1 \\
\hline
\end{tabular}

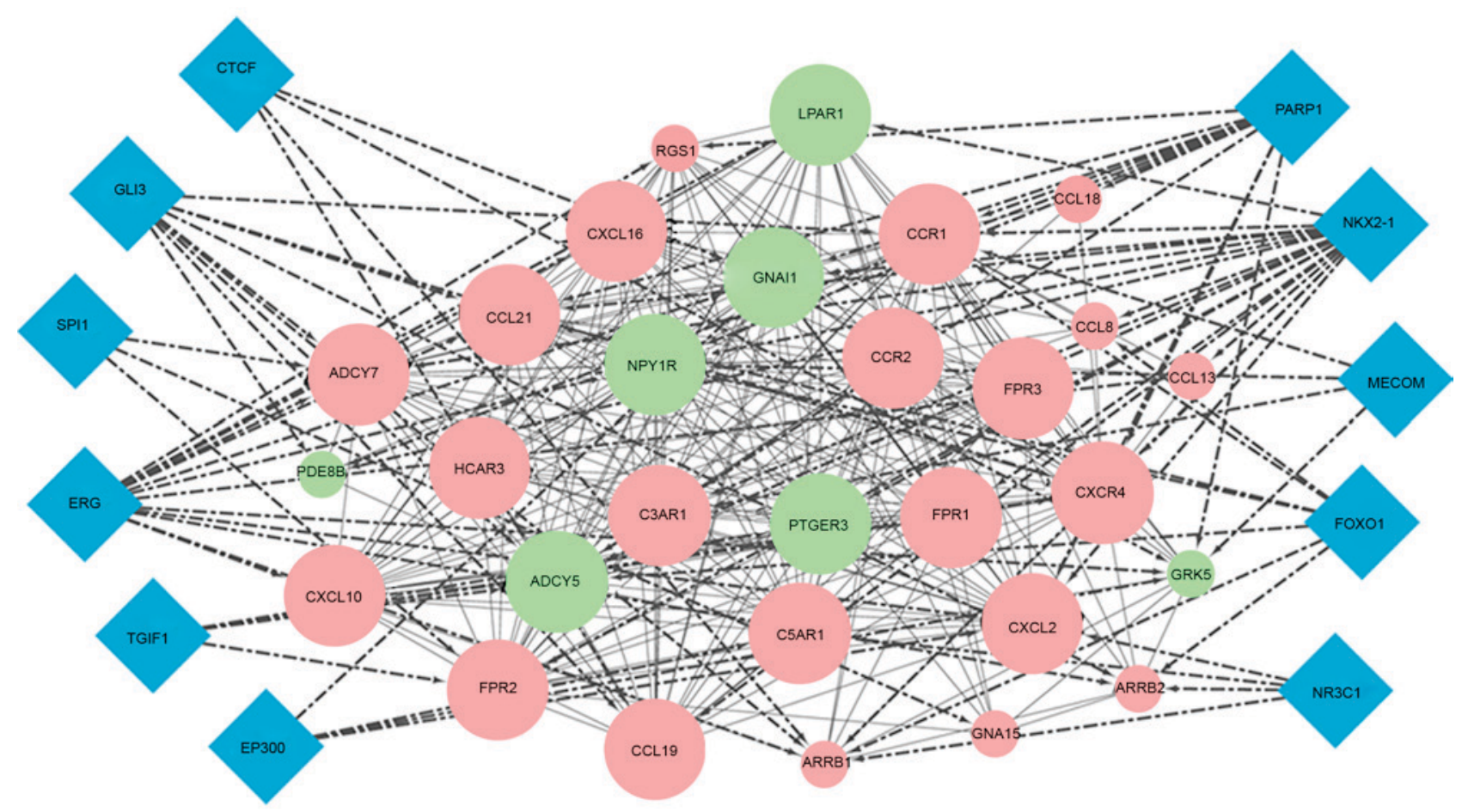

Figure 7. Significant modules in the protein-protein interaction network and predicted transcription factors targeting to genes in this module. The red and green circles represent the upregulated and downregulated genes identified in the study, respectively. The blue rhombi represent transcription factors. The dotted lines indicate the regulatory relationship between transcription factors and target genes. The solid grey line indicates the interaction between target genes.

carotid atheroma plaque samples and macroscopically intact tissue samples adjacent to the atheroma plaque from the same hypertensive patient, and as a key nodes in the PPI network constructed with using DEGs between these two groups. Therefore, this gene may be an essential marker in predicting plaque formation within the carotid artery.

PIK3CG encodes an enzyme, which can phosphorylate phosphoinositides. Single nucleotide polymorphisms (SNPs) close to or within PIK3CG have been shown to have important clinical significance. For example, the rs342286 SNP, located $140 \mathrm{~kb}$ upstream of the PIK3CG gene, may be associated with increased platelet aggregation and acute coronary syndromes (29). A genome-wide association study by Wain et al (30) on pulse pressure and mean arterial pressure demonstrated $7 \mathrm{q} 22.3$ close to PIK3CG as a novel pulse pressure locus, which may affect systolic blood pressure and diastolic blood pressure. In carotid artery plaques, a combined meta-analysis confirmed rs17398575, situated $96.5 \mathrm{~kb}$ from the PIK3CG gene, as the most significant signal, which may induce an $18 \%$ increase in chance of plaque presence (31), although other reports do not support the association of this locus with subclinical atherosclerosis (32). The results of the present study showed that $P I K 3 C G$ was upregulated in carotid atheroma plaque samples, compared with macroscopically intact tissue samples from the same individual. It was also a key node in the PPI network with a relatively high degree. This suggested PIK3CG as one of key genes associated with the formation of carotid plaques.

Taken together, the DEGs identified in the carotid atheroma plaque samples, when compared with macroscopically intact tissue samples, may be involved in carotid atherogenesis. The key nodes identified in the PPI network constructed with these DEGs and genes involved in the significant module, including $S Y K, L Y N$ and $P I K 3 C G$, are 
promising for the prediction of carotid plaque formation. Further experimental evidence is required to confirm these findings.

\section{References}

1. Fatahzadeh M and Glick M: Stroke: Epidemiology, classification risk factors, complications, diagnosis, prevention and medical and dental management. Oral Surg Oral Med Oral Pathol Oral Radiol Endod 102: 180-191, 2006.

2. Friedlander AH and Baker JD: Panoramic radiography: An aid in detecting patients at risk of cerebrovascular accident. J Am Dent Assoc 125: 1598-1603, 1994.

3. Foulkes MA, Wolf PA, Price TR, Mohr JP and Hier DB: The Stroke data bank: Design, methods, and baseline characteristics Stroke 19: 547-554, 1988

4. Beckstrom BW, Horsley SH, Scheetz JP, Khan Z, Silveira AM, Clark SJ, Greenwell H and Farman AG: Correlation between carotid area calcifications and periodontitis: A retrospective study of digital panoramic radiographic findings in pretreatment cancer patients. Oral Surg Oral Med Oral Pathol Oral Radiol Endod 103: 359-366, 2007.

5. Kappelle LJ: Symptomatic carotid artery stenosis. J Neurol 249: 254-259, 2002.

6. Ayari H and Bricca G: Identification of two genes potentially associated in iron-heme homeostasis in human carotid plaque using microarray analysis. J Biosci 38: 311-315, 2013.

7. Barrett T, Wilhite SE, Ledoux P, Evangelista C, Kim IF, Tomashevsky M, Marshall KA, Phillippy KH, Sherman PM, Holko M, et al: NCBI GEO: Archive for functional genomics data sets-update. Nucleic Acids Res 41 (Database issue): D991-D995, 2013.

8. Stary HC, Chandler AB, Dinsmore RE, Fuster V, Glagov S, Insull W Jr, Rosenfeld ME, Schwartz CJ, Wagner WD and Wissler RW: A definition of advanced types of atherosclerotic lesions and a histological classification of atherosclerosis. A report from the committee on vascular lesions of the council on arteriosclerosis, American heart association. Arterioscler Thromb Vasc Biol 15: 1512-1531, 1995.

9. Carvalho BS and Irizarry RA: A framework for oligonucleotide microarray preprocessing. Bioinformatics 26: 2363-2367, 2010.

10. Ritchie ME, Phipson B, Wu D, Hu Y, Law CW, Shi W and Smyth GK: limma powers differential expression analyses for RNA-sequencing and microarray studies. Nucleic Acids Res 43: e47, 2015.

11. Benjamini Y, Drai D, Elmer G, Kafkafi N and Golani I: Controlling the false discovery rate in behavior genetics research. Behav Brain Res 125: 279-284, 2001.

12. Diboun I, Wernisch L, Orengo CA and Koltzenburg M: Microarray analysis after RNA amplification can detect pronounced differences in gene expression using limma. BMC Genomics 7: 252, 2006.

13. Huang DW, Sherman BT, Tan Q, Collins JR, Alvord WG, Roayaei J, Stephens R, Baseler MW, Lane HC and Lempicki RA: The DAVID gene functional classification tool: A novel biological module-centric algorithm to functionally analyze large gene lists. Genome Biol 8: R183, 2007.

14. Ashburner M, Ball CA, Blake JA, Botstein D, Butler H, Cherry JM, Davis AP, Dolinski K, Dwight SS, Eppig JT, et al: Gene ontology: Tool for the unification of biology. The gene ontology consortium. Nat Genet 25: 25-29, 2000.

15. Kanehisa M, Araki M, Goto S, Hattori M, Hirakawa M, Itoh M, Katayama T, Kawashima S, Okuda S, Tokimatsu T and Yamanishi Y: KEGG for linking genomes to life and the environment. Nucleic Acids Res 36 (Database issue): D480-D484, 2008.

16. Szklarczyk D, Franceschini A, Wyder S, Forslund K, Heller D, Huerta-Cepas J, Simonovic M, Roth A, Santos A, Tsafou KP, et al: STRING v10: Protein-protein interaction networks, integrated over the tree of life. Nucleic Acids Res 43 (Database issue): D447-D452, 2015.
17. Smoot ME, Ono K, Ruscheinski J, Wang PL and Ideker T: Cytoscape 2.8: New features for data integration and network visualization. Bioinformatics 27: 431-432, 2011.

18. Janky R, Verfaillie A, Imrichová H, Van de Sande B, Standaert L, Christiaens V, Hulselmans G, Herten K, Naval Sanchez M, Potier D, et al: iRegulon: From a gene list to a gene regulatory network using large motif and track collections. PLoS Comput Biol 10: e1003731, 2014

19. Koton S, Schneider AL, Rosamond WD, Shahar E, Sang Y, Gottesman RF and Coresh J: Stroke incidence and mortality trends in US communities, 1987 to 2011. JAMA 312: 259-268, 2014.

20. Wakhloo AK, Lieber BB, Seong J, Sadasivan C, Gounis MJ, Miskolczi L and Sandhu JS: Hemodynamics of carotid artery atherosclerotic occlusive disease. J Vasc Interv Radiol 15: S111-S121, 2004

21. Attila M, Jürgen R and Tybulewicz VL: The SYK tyrosine kinase: A crucial player in diverse biological functions. Nat Rev Immunol 10: 387-402, 2010.

22. Mallat Z, Hugel B, Ohan J, Lesèche G, Freyssinet JM and Tedgui A: Shed membrane microparticles with procoagulant potential in human atherosclerotic plaques: A role for apoptosis in plaque thrombogenicity. Circulation 99: 348-353, 1999.

23. Ait-Oufella H, Pouresmail V, Simon T, Blanc-Brude O, Kinugawa K, Merval R, Offenstadt G, Lesèche G, Cohen PL, Tedgui A and Mallat Z: Defective mer receptor tyrosine kinase signaling in bone marrow cells promotes apoptotic cell accumulation and accelerates atherosclerosis. Arterioscler Thromb Vasc Biol 28: 1429-1431, 2008

24. Choi SH, Yin H, Ravandi A, Armando A, Dumlao D, Kim J, Almazan F, Taylor AM, McNamara CA, Tsimikas S, et al: Polyoxygenated cholesterol ester hydroperoxide activates TLR4 and SYK dependent signaling in macrophages. PLoS One 8: e83145, 2013.

25. Hilgendorf I, Eisele S, Remer I, Schmitz J, Zeschky K, Colberg C, Stachon P, Wolf D, Willecke F, Buchner M, et al: The oral spleen tyrosine kinase inhibitor fostamatinib attenuates inflammation and atherogenesis in low-density lipoprotein receptor-deficient mice. Arterioscler Thromb Vasc Biol 31: 1991-1999, 2011

26. Saito YD, Jensen AR, Salgia R and Posadas EM: Fyn: A novel molecular target in cancer. Cancer 116: 1629-1637, 2010.

27. Toubiana J, Rossi A-L,Belaidouni N, Grimaldi D,Pene F, Chafey P, Comba B, Camoin L, Bismuth G, et al: Src-family-tyrosine kinase Lyn is critical for TLR2-mediated NF- $\kappa \mathrm{B}$ activation through the PI 3-kinase signaling pathway. Innate immunity 21 : 685-697, 2015.

28. Horiuchi M: Anti-inflammatory effect of nifedipine and vasculoprotection. Drugs 66: 28-30, 2006

29. Sawczuk M, Maciejewska-Karłowska A, Skotarczak B and Pawlik A: Association between single nucleotide polymorphism rs342286 near the PIK3CG gene and acute coronary syndromes. Pol Arch Med Wewn 124: 210-212, 2014.

30. Wain LV, Verwoert GC, O'Reilly PF, Shi G, Johnson T, Johnson AD, Bochud M, Rice KM, Henneman P, Smith AV, et al: Genome-wide association study identifies six new loci influencing pulse pressure and mean arterial pressure. Nat Genet 43: 1005-1011, 2011.

31. Bis JC, Kavousi M, Franceschini N, Isaacs A, Abecasis GR, Schminke U, Post WS, Smith AV, Cupples LA, Markus HS, et al: Meta-analysis of genome-wide association studies from the CHARGE consortium identifies common variants associated with carotid intima media thickness and plaque. Nat Genet 43: 940-947, 2011

32. Adams JN, Raffield LM, Freedman BI, Langefeld CD, Ng MC, Carr JJ, Cox AJ and Bowden DW: Analysis of common and coding variants with cardiovascular disease in the diabetes heart study. Cardiovasc Diabetol 13: 77, 2014. 